Check for updates

Cite this: RSC Adv., 2017, 7, 26074

\title{
Concentration-dependent self-assembly structures of an amphiphilic perylene diimide with tri(ethylene glycol) substituents at bay positions $\uparrow$
}

\author{
Xin Wang, $\dot{t}^{\mathrm{a}}$ Ting Zeng, $\dot{+}^{\mathrm{a}}$ Mohamed Nourrein, ${ }^{\mathrm{a}}$ Bo-Han Lai, ${ }^{\mathrm{b}}$ Kaiwen Shen, ${ }^{\mathrm{a}}$ \\ Chien-Lung Wang, ${ }^{* b}$ Bin Sun (D) *a and Meifang Zhu*a
}

\begin{abstract}
In this work, an amphiphilic perylene diimide (1,7-TEG-PDI- $\left.\mathrm{C}_{12}\right)$ that bears two hydrophilic triethylene glycol (TEG) chains at its bay position, and two hydrophobic dodecyl chains at its imide position was synthesized to identify the roles of concentration and $\mathrm{H}$-bonding on the self-assembly morphology of the amphiphilic PDI. Since 1,7-TEG-PDI- $C_{12}$ was prepared from the reaction of two bifunctional reactants, TEG and $N, N^{\prime}-\operatorname{bis}(n-$ dodecyl)-1,7-dibromo-perylene-3,4:9,10-tetracarboxylic diimide, careful choices of solvent, base, and the stoichiometry of crown ether and base were found to be critical in reaching high reaction yield under mild conditions. TEM and SEM results revealed the abundant concentration-dependent self-assembly morphologies of 1,7-TEG-PDI- $\mathrm{C}_{12}$. Characterization results including UV-vis, fluorescence, NMR, IR and $\mathrm{XRD}$ analysis show that the formation of the self-assembled structure is a synergetic result of the intermolecular $\pi-\pi$ interaction and $\mathrm{H}$-bonding of 1,7-TEG-PDI- $\mathrm{C}_{12}$.
\end{abstract}

Received 17th April 2017

Accepted 29th April 2017

DOI: 10.1039/c7ra04296e

rsc.li/rsc-advances

Self-assembly of amphiphilic molecules is usually conducted

\section{Introduction}

Over the years, supramolecular self-assembly has attracted increasing attention as an interdisciplinary breakthrough research field in nanotechnology and biological sciences. ${ }^{1}$ In consideration of their potential applications in diverse fields, supramolecular self-assembly exploits non-covalent interactions between molecules to form miscellaneous architectures. ${ }^{2}$ Moreover, it offers an attractive pathway to construct wellorganized functional nanomaterials, which help bridge the gap between natural and artificial systems. ${ }^{3}$

These nanostructures are cooperatively controlled by noncovalent forces including $\mathrm{H}$-bonding, dipole-dipole attraction, $\pi-\pi$ stacking, van der Waals force, hydrophobic effect, electrostatic interaction, and metal-ligand coordination. ${ }^{4}$ Under the multiple interactions, advanced materials built by supramolecular self-assembly have made some effective progress. ${ }^{3}$ Shape, size, and intermolecular interactions of the individual building blocks are important factors which drive the selfassembly process toward their supramolecular architecture..$^{5-9}$

\footnotetext{
${ }^{a}$ State Key Laboratory for Modification of Chemical Fibers and Polymer Materials, College of Materials Science and Engineering, Center for Advanced Low-dimension Materials, Donghua University, 201620, Shanghai, P. R. China. E-mail: sunbin@ dhu.edu.cn; zmf@dhu.edu.cn

${ }^{b}$ Department of Applied Chemistry, National Chiao Tung University, 1001 Ta Hsueh Road, Hsin-Chu, 30010, Taiwan. E-mail: kclwang@nctu.edu.tw

$\dagger$ Electronic supplementary information (ESI) available: The synthetic details, ${ }^{1} \mathrm{H}$ NMR, ${ }^{13} \mathrm{C}$ NMR and mass spectra of 1,7 -TEG-PDI-C ${ }_{12}$ are available. See DOI: 10.1039/c7ra04296e

$\$$ These authors contribute equally to the work.
} in the solution phase and is governed by weak non-covalent interactions, which is a fundamental process not only in nature but also in supra-molecular chemistry. ${ }^{1}$ Despite many efforts, self-assembly of amphiphilic molecules to form complex functional architectures by multi-physical interaction (the synergistic effects of the $\pi-\pi$ stacking, hydrophobic effect and the hydrogen bond interaction) represents still a very challenging and unsolved task..$^{10}$ Moreover, adjustable and controllable ordered structure by multi-physical interaction certainly merit additional study with novel optical and electronic functionalities. ${ }^{11}$

As good building blocks for self-organized molecular materials with highly ordered structure, perylene diimides (PDI) attract a great interest owing to their applications in diverse fields of physical organic chemistry. ${ }^{4}$ Up to now, they were widely studied as n-type semiconductors and attracted increasing attention due to their wide optical absorption in the visible to near-infrared spectral region and significant charge transport properties. Therefore, PDI-based materials have been used as prominent chromophores in the fluorescence probe, field effect transistor, ${ }^{12}$ molecular electronics, solar cells and emitting diodes and other fields owing to their unique spectroscopic properties and extraordinary photostability. ${ }^{13}$

To achieve better control of the steerable spatial organization of PDIs in solid-state or supramolecular aggregates, significant efforts have been dedicated to develop new amphiphilic PDI derivatives over the past few years. ${ }^{14,15}$ Based on their molecular structures, the current amphiphilic PDI derivatives can be largely divided into two groups. In the first group, the PDI derivatives have one hydrophobic segment and one 
hydrophilic segment at the two imide positions of the molecule. In the second group, units with different hydrophilicity are placed at the imide positions and the bay positions of a PDI derivative, respectively. Würthner and coworkers synthesized the wedge- and dumbbell-shaped amphiphilic PDIs ${ }^{\mathbf{1 6}}$ and reported the first example on the morphological control of PDI nanoaggregates by co-assembly of the two PDI derivatives in aqueous solution. Yao and co-workers ${ }^{\mathbf{1 7}}$ synthesized an amphiphilic PDI that has two pyridyloxyl groups at the bay position and manipulated the self-assembly morphology of the PDI in solution via changing the protonation state of the pyridyl groups. Baram et al. synthesized an amphiphilic bis-PDI derivative that bears two poly(ethylene oxide) chains at the bay position and manipulated the self-assembly morphology via reversible charging of the bisPDI derivative. ${ }^{15}$ Although solution concentration has been known as an important factor in the self-assembly morphology of amphiphilic molecules, its role in the self-assembly of amphiphilic PDIs was not clearly identified in the previous studies, because the roles of concentration were concealed by the more dominating factors such as molecular geometry, ionic interactions, and oxidation states of the PDI molecules.

To reveal the influences of solution concentration on the self-assembly morphology of amphiphilic PDI, in this work, a novel amphiphilic PDI (1,7-TEG-PDI-C 12 ) that bear two hydrophilic triethylene glycol (TEG) chains at its bay position, and two hydrophobic dodecyl chains at its imide position was synthesized according to Scheme 1 . Hydroxyl group was kept as the end-group of the TEG segment to introduce H-bond interaction along the lateral direction of the PDI molecule. Careful choices of solvent, base and the stoichiometries of crown ether and base were found to be critical to reach high yield under mild reaction conditions and to avoid polymerization. The change of aggregation states of 1,7-TEG-PDI-C $\mathrm{C}_{12}$ was investigated by UV-vis and fluorescence (FL) spectroscopy, and the concentration-dependent self-assembly morphologies of 1,7TEG-PDI- $\mathrm{C}_{12}$ were studied by scanning electron microscopy (SEM), transmission electron microscopy (TEM), and X-ray diffraction (XRD) analysis. The characterization results reveal the important role of concentration and the bay-position - $\mathrm{OH}$ groups in the abundant self-assembly morphologies of amphiphilic PDI molecules.
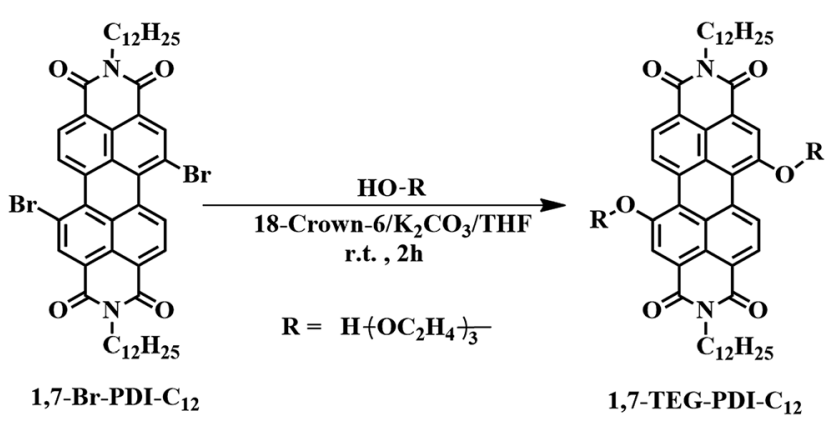

1,7-TEG-PDI-C 12

Scheme 1 Synthetic conditions of $N, N^{\prime}$-bis(n-dodecyl)-1,7-di(triethylene glycol)-perylene-3,4,9,10-tetracarboxyl-diimide (1,7-TEG$\left.\mathrm{PDI}-\mathrm{C}_{12}\right)$. N, $N^{\prime}$-Bis(n-dodecyl)-1,7-dibromo-perylene-3,4:9,10-tetracar-boxylic diimide (1,7-Br-PDI- $\left.\mathrm{C}_{12}\right)$ was synthesized according to the literature..$^{18,19}$

\section{Results and discussion}

\section{Molecular design and synthesis}

The synthetic scheme of 1,7-TEG-PDI-C ${ }_{12}$ is shown in Scheme 1. Although 1,7-disubstituted amphiphilic PDI derivatives have been reported in several works, ${ }^{15,20-23}$ adding bifunctional substituent, such as TEG, onto 1,7-dibrominated PDI remains unexplored. Because both 1,7-dibrominated PDI and TEG are bifunctional, if the reaction conditions are not optimized, oligo(PDI)s or even poly(PDI) may be generated in the reaction, resulting in the low yield of $1,7-\mathrm{TEG}-\mathrm{PDI}-\mathrm{C}_{12}$. To produce $1,7-$ TEG-PDI- $\mathrm{C}_{12}$ in high yield, an excess amount of TEG (4.5 equiv. to $1,7-\mathrm{Br}-\mathrm{PDI}-\mathrm{C}_{12}$ ) were used, and the reaction conditions were optimized.

The influences of solvent, alkali, crown ether and reaction time on the yield of 1,7-TEG-PDI- $\mathrm{C}_{12}$ are summarized shown in Table 1 . Due to the low solubility of alkali in organic solvent, the reactions using $\mathrm{CHCl}_{3}$ as a solvent or without the addition of 18crown- 6 did not proceed. The increase of the equiv. of 18-crown6 or $\mathrm{K}_{2} \mathrm{CO}_{3}$ (with respect to the equiv. of 1,7-Br-PDI-C ${ }_{12}$ ) improves the reaction yield, as can be seen in entry 8-11. The highest reaction yield of $80 \%$ was obtained in entry 12 . Comparing the yields of entry 11 and entry 12, the reaction gave almost identical yield at two hours and twelve hours, suggesting the reaction can be completed at room temperature in two hours. The reaction is also sensitive to the basicity of alkali. Entry 4 and entry 5 show that alkali with basicity stronger or weaker than $\mathrm{K}_{2} \mathrm{CO}_{3}$ gave lower reaction yield. Changing the alkali from $\mathrm{K}_{2} \mathrm{CO}_{3}$ to $\mathrm{Na}_{2} \mathrm{CO}_{3}$ (entry 7) also decreased the yield, because 18-crown-6 has a better affinity for potassium cations than sodium cations. ${ }^{1} \mathrm{H}$ NMR, ${ }^{13} \mathrm{C}$ NMR and MALDI-TOF-MS spectra in Fig. S1-S3† confirm the chemical identity of 1,7TEG-PDI- $\mathrm{C}_{12}$ and the success of the reaction.

\section{Photophysical properties of 1,7-TEG-PDI- $\mathrm{C}_{12}$ in solutions}

UV-Vis and FL spectra of PDI have been widely used to study the PDI aggregations in the solution phases, due to the sensibility of the spectra to the chromophoric group distance, orientation and therefore. ${ }^{21}$ Fig. 1a shows the UV-visible absorption spectra of 1,7-TEG-PDI-C 12 in $\mathrm{CHCl}_{3}$ at different concentrations. The

Table 1 Reaction conditions for the preparation of 1,7-TEG-PDI- $\mathrm{C}_{12}$

\begin{tabular}{|c|c|c|c|c|c|}
\hline Entry & $\begin{array}{l}\text { 18-Crown-6 } \\
\text { (equiv.) }\end{array}$ & Alkali (equiv.) & Solvent & Time $[\mathrm{h}]$ & Yield [\%] \\
\hline 1 & 1.0 & None & THF & 12 & 0 \\
\hline 2 & None & $\mathrm{K}_{2} \mathrm{CO}_{3}(4.0)$ & THF & 12 & 0 \\
\hline 3 & 4.0 & $\mathrm{~K}_{2} \mathrm{CO}_{3}(4.0)$ & $\mathrm{CHCl}_{3}$ & 12 & 0 \\
\hline 4 & 4.0 & $\mathrm{KHCO}_{3}(4.0)$ & THF & 2 & 18 \\
\hline 5 & 4.0 & $\mathrm{KOH}(4.0)$ & THF & 2 & 20 \\
\hline 6 & 1.0 & $\mathrm{~K}_{2} \mathrm{CO}_{3}(1.0)$ & THF & 12 & 45 \\
\hline 7 & 4.0 & $\mathrm{Na}_{2} \mathrm{CO}_{3}(4.0)$ & THF & 2 & 57 \\
\hline 8 & 1.0 & $\mathrm{~K}_{2} \mathrm{CO}_{3}(2.0)$ & THF & 12 & 73 \\
\hline 9 & 1.0 & $\mathrm{~K}_{2} \mathrm{CO}_{3}(4.0)$ & THF & 12 & 75 \\
\hline 10 & 2.0 & $\mathrm{~K}_{2} \mathrm{CO}_{3}(4.0)$ & THF & 12 & 77 \\
\hline 11 & 4.0 & $\mathrm{~K}_{2} \mathrm{CO}_{3}(4.0)$ & THF & 12 & 79 \\
\hline 12 & 4.0 & $\mathrm{~K}_{2} \mathrm{CO}_{3}(4.0)$ & THF & 2 & 80 \\
\hline
\end{tabular}



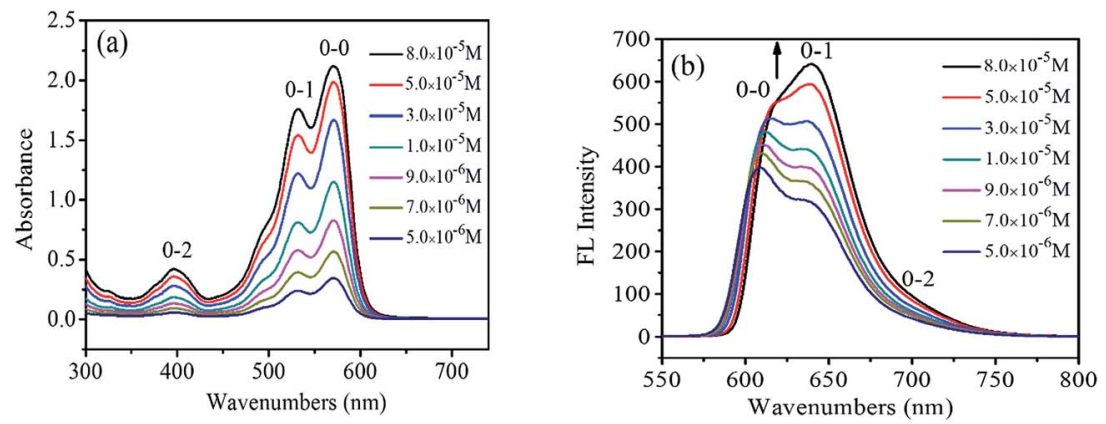

Fig. 1 (a) UV-vis absorption spectra and (b) FL spectra of 1,7-TEG-PDI- $\mathrm{C}_{12}$ in $\mathrm{CHCl}_{3}$ at different concentrations.

absorbance of 1,7-TEG-PDI-C ${ }_{12}$ increases with the increases of solution concentration. The characteristic absorption peaks of PDI appeared at 570, 530 and $492 \mathrm{~nm}$ due to $\pi-\pi *$ electronic transitions, which represent three electronic vibration levels of $0-0,0-1$ and $0-2$, respectively. The absorption peak at $395 \mathrm{~nm}$ is derived from the $n-\pi *$ transition between $n$ electrons of oxygen atom and $\pi$-orbital of carbon-carbon double bond of triethylene glycol.

The FL spectra of 1,7-TEG-PDI- $\mathrm{C}_{12}$ in $\mathrm{CHCl}_{3}$ at different concentrations were recorded in Fig. 1b. At the lower concentrations $\left(5.0 \times 10^{-6}\right.$ to $\left.3.0 \times 10^{-5} \mathrm{~mol} \mathrm{~L}^{-1}\right)$, the $\lambda_{\max }$ of FL was located at around $604 \mathrm{~nm}$, corresponding to the $0-0$ vibronic transition. As the concentration increases, the $0-1$ vibronic transition at $639 \mathrm{~nm}$ ascends and exceeds the level of $0-0$, becoming the main emission peak and following with the gradually red shift in the spectrum. The results suggest that the increase of solution concentration increases the proportion of PDI aggregates in the solution, resulting in the decrease of monomolecular emission and the increase of the aggregation emission.

The UV-vis adsorption spectra of 1,7-TEG-PDI- $\mathrm{C}_{12}$ in hexanol are shown in Fig. 2. The positions of the adsorption bands are similar to those of $1,7-$ TEG-PDI-C ${ }_{12}$ in $\mathrm{CHCl}_{3}$. Noteworthy, as the solution concentration increases, the absorbance of the 0-1 vibronic transition gradually becomes higher than that of the $0-$ 0 vibronic transition. It is an indication of strong $\pi-\pi$ interaction between the molecules, which reflects the aggregation of 1,7-TEG-PDI-C ${ }_{12}$. In $\mathrm{CHCl}_{3}$, the absorbance of the $0-0$ vibronic transition is always higher than that of the $0-1$ transition. Since hexanol has higher polarity than $\mathrm{CHCl}_{3}$, the different concentration dependence thus indicates that aggregation of 1,7-TEG-PDI- $\mathrm{C}_{12}$ can be promoted by the increase of solvent polarity. The FL spectra of 1,7-TEG-PDI- $\mathrm{C}_{12}$ in hexanol are shown in Fig. 2b. The increase of the solution concentration causes great changes in the shape, intensity and the location of the emission band. Fig. $2 \mathrm{~b}$ and $\mathrm{c}$ are used to emphasize the changes in the emission spectra of 1,7-TEG-PDI- $\mathrm{C}_{12}$ at the higher concentrations $\left(3.0 \times 10^{-6}\right.$ to $\left.1.0 \times 10^{-4} \mathrm{~mol} \mathrm{~L}^{-1}\right)$, and at the lower concentrations $\left(9.0 \times 10^{-8}\right.$ to $\left.3.0 \times 10^{-6} \mathrm{~mol} \mathrm{~L}^{-1}\right)$, respectively. The FL intensity of 1,7-TEG-PDI- $\mathrm{C}_{12}$ increases first with solution concentration from $9.0 \times 10^{-8}$ to $3.0 \times 10^{-6} \mathrm{~mol}$ $\mathrm{L}^{-1}$, but above $3.0 \times 10^{-6} \mathrm{~mol} \mathrm{~L}^{-1}$, the further increase of solution concentration decreases the FL intensity. Moreover, the intensity of the $0-0$ emission decreases significantly with the increase of solution concentration. At $1.0 \times 10^{-4} \mathrm{~mol} \mathrm{~L}^{-1}$, the $0-0$ emission almost disappeared, and the unimodal FL band, which has a significant red shift was observed. The quench of the FL occurs because the short-wavelength FL band $(535 \mathrm{~nm})$ overlapped with the absorption band of 1,7-TEG-PDI$\mathrm{C}_{12}$ at $527 \mathrm{~nm}$. At the higher concentrations, self-quenching of FL becomes more significant and decreases the FL intensity. Moreover, 1,7-TEG-PDI-C ${ }_{12}$ molecules aggregate at high solution concentration. Since the FL intensity of aggregates are weaker, a decline in the overall FL intensity was observed. ${ }^{24}$

\section{Solvent, concentration and temperature effects on the 1,7- TEG-PDI-C $\mathrm{C}_{22}$ aggregates}

Fig. 3a shows the normalized UV absorption spectra of the hexanol solution and the $\mathrm{CHCl}_{3}$ solution of 1,7-TEG-PDI- $\mathrm{C}_{12}$ at the same concentration $\left(8.0 \times 10^{-5} \mathrm{~mol} \mathrm{~L}^{-1}\right)$. The ratio of the
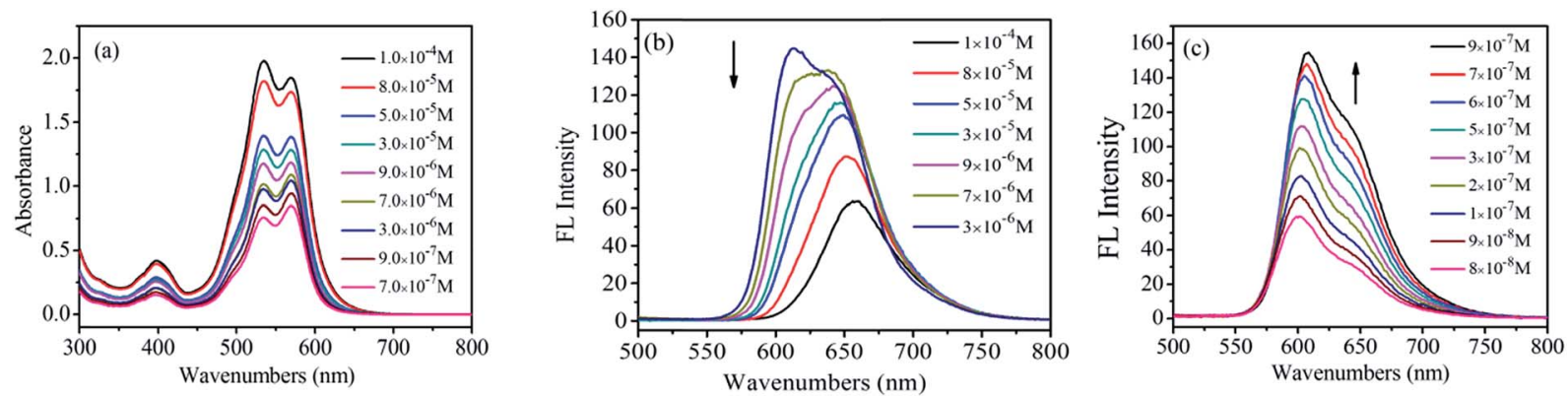

Fig. 2 (a) UV-visible absorption spectra, and (b), (c) FL emission spectra of 1,7-TEG-PDI- $\mathrm{C}_{12}$ in hexanol at different concentrations. (b and c) Emphasize the FL emission spectra of 1,7-TEG-PDI- $\mathrm{C}_{12}$ at the higher concentrations, and the lower concentrations, respectively. 

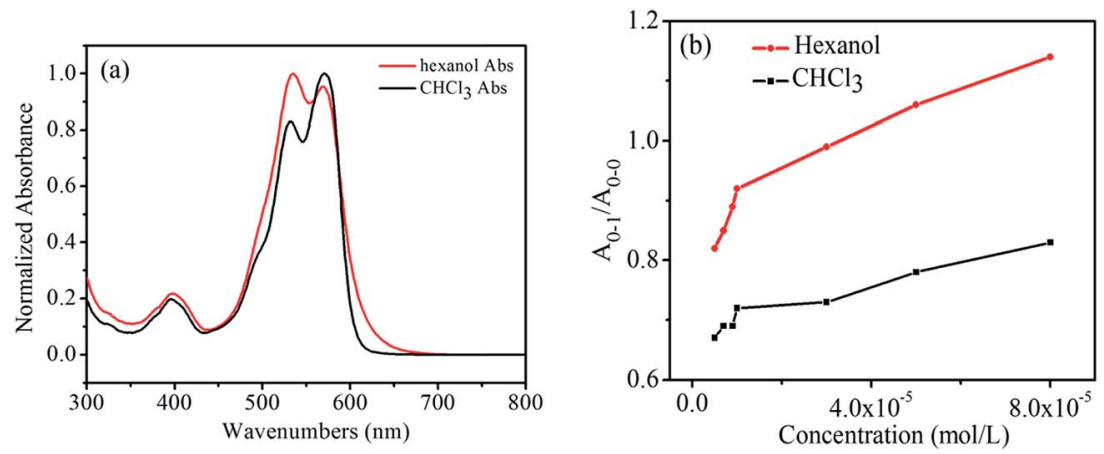

Fig. 3 (a) Normalized UV-visible absorption spectra of 1,7-TEG-PDI- $\mathrm{C}_{12}$ at $8.0 \times 10^{-5} \mathrm{~mol} \mathrm{~L}^{-1}$; (b) the $A_{0-1} / A_{0-0}$ ratios of $1,7-\mathrm{TEG}-\mathrm{PDI}-\mathrm{C}_{12}$ at different concentrations in $\mathrm{CHCl}_{3}$ and hexanol.

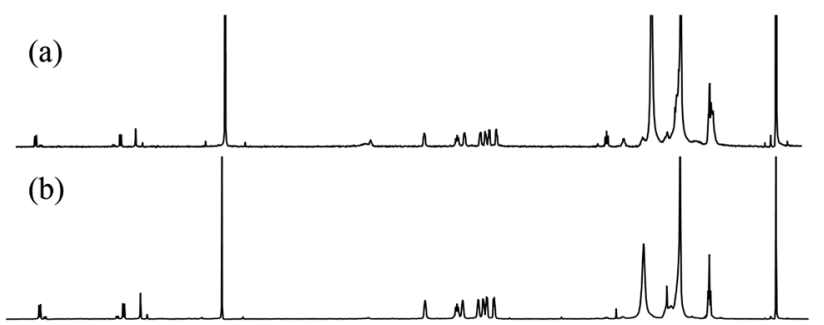

(c)

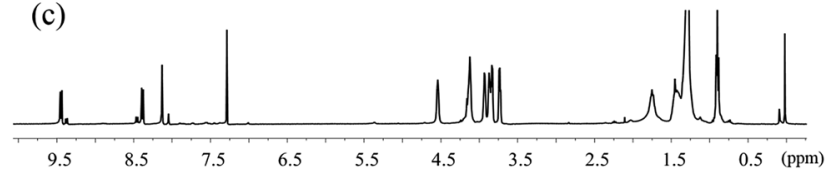

Fig. $4{ }^{1} \mathrm{H}$-NMR spectra of 1,7-TEG-PDI- $\mathrm{C}_{12}$ in $\mathrm{CDCl}_{3}$ at concentrations of (a) $1.0 \times 10^{-5} \mathrm{~mol} \mathrm{~L}^{-1}$, (b) $5.0 \times 10^{-5} \mathrm{~mol} \mathrm{~L}^{-1}$, and (c) $1.0 \times$ $10^{-4} \mathrm{~mol} \mathrm{~L}^{-1}$.

absorption maxima of the $0-1$ vibronic transition $\left(A_{0-1}\right)$ and the absorption maxima of the $0-0$ vibronic transition $\left(A_{0-0}\right)$, i.e. $A_{0-1} /$ $A_{0-0}$, is used to qualitatively evaluate the proximity of the PDI units in solution. As indicated by Fimmel et al. ${ }^{25}$ PDI foldamers in the folded state give a higher $A_{0-1} / A_{0-0}$ ratio than those in the unfolded state. In other words, a closer PDI aggregate in solution leads to the increase of the $A_{0-1} / A_{0-0}$ ratio. Accordingly, because in Fig. $3 \mathrm{a}$, the $A_{0-1} / A_{0-0}$ ratio of $1,7-\mathrm{TEG}-\mathrm{PDI}-\mathrm{C}_{12}$ is larger in hexanol than it is in $\mathrm{CHCl}_{3}$, it can be found that hexanol promotes the aggregate of $1,7-\mathrm{TEG}$-PDI-C ${ }_{12}$ more than $\mathrm{CHCl}_{3}$ does.

In Fig. $3 \mathrm{~b}$, the $A_{0-1} / A_{0-0}$ ratio of the hexanol and $\mathrm{CHCl}_{3}$ solutions of 1,7-TEG-PDI-C ${ }_{12}$ also increases as the solution concentration increases. Besides, in the ${ }^{1} \mathrm{H}-\mathrm{NMR}$ spectra (Fig. 4), the proton signals of the perylene core (at 10.0-8.0 ppm) shows clear upfield shifts as the solution concentration increases. The upfield shift of these protons also supports that the increased solution concentration promotes a more proximal packing of 1,7-TEG-PDI-C ${ }_{12}$ molecules in solution. Based on the UV-vis and ${ }^{1} \mathrm{H}$-NMR results, it is evident that 1,7-TEG-PDI- $\mathrm{C}_{12}$ molecules are more strongly aggregate, and experience stronger intermolecular interactions at higher solution concentrations.

To study how temperature affects the aggregates, the absorption spectra of 1,7-TEG-PDI-C ${ }_{12}$ solution in hexanol (8.0 $\times 10^{-6} \mathrm{~mol} \mathrm{~L}^{-1}$ ) were measured at temperatures from $20{ }^{\circ} \mathrm{C}$ to $75{ }^{\circ} \mathrm{C}$. As shown in Fig. 5a, the increase of solution temperature decreases the $A_{0-1} / A_{0-0}$ ratio of the 1,7-TEG-PDI-C ${ }_{12}$ solution. Fig. $5 \mathrm{~b}$ schematically displays the temperature dependence of the $A_{0-1} / A_{0-0}$ ratios. The increase of the thermal energy thus interrupts the intermolecular interaction and disrupts aggregate state of 1,7-TEG-PDI-C ${ }_{12}$.

\section{Self-assembled morphologies of 1,7-TEG-PDI-C $\mathrm{C}_{12}$ via rapid solution mixing method}

In the previous sections, the UV-vis and ${ }^{1} \mathrm{H}$ NMR results have shown that 1,7-TEG-PDI-C 12 molecules aggregate differently at
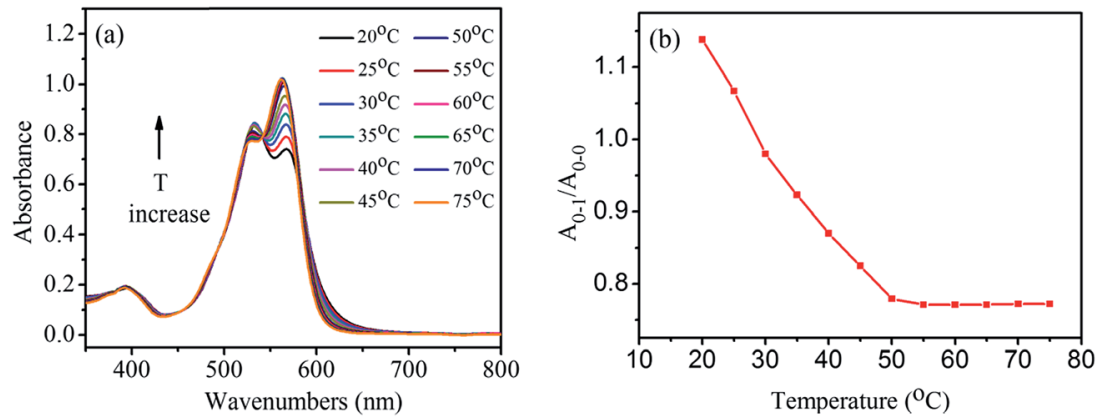

Fig. 5 (a) The UV-visible absorption spectra of the hexanol solution of $1,7-\mathrm{TEG}$-PDI- $\mathrm{C}_{12}\left(8.0 \times 10^{-6} \mathrm{~mol} \mathrm{~L}^{-1}\right)$ at temperatures from $20^{\circ} \mathrm{C}$ to $75^{\circ} \mathrm{C}$; (b) the temperature dependence of the $A_{0-1} / A_{0-0}$ ratio of the solution. 
different solution concentrations. To study the influences of solution concentration on the self-assembled morphology of 1,7-TEG-PDI-C ${ }_{12}, 100 \mu \mathrm{L}$ of the THF solutions of 1,7-TEG-PDI$\mathrm{C}_{12}$ at concentrations of $1 \times 10^{-5} \mathrm{~mol} \mathrm{~L}^{-1}, 1 \times 10^{-4} \mathrm{~mol} \mathrm{~L}^{-1}$, and $1 \times 10^{-3} \mathrm{~mol} \mathrm{~L}^{-1}$ were directly mixed with $3 \mathrm{~mL}$ of distilled water to reach the volume ratio of $V_{\mathrm{THF}} / V_{\text {water }}=1 / 30$, and then balanced under room temperature for 24 hours. In Fig. 6, the different $A_{0-1} / A_{0-0}$ ratios of the THF solutions of 1,7-TEG-PDI$\mathrm{C}_{12}$ indicate that 1,7-TEG-PDI- $\mathrm{C}_{12}$ molecules are at different aggregation states at the concentrations of $1 \times 10^{-5} \mathrm{~mol} \mathrm{~L}^{-1}, 1$ $\times 10^{-4} \mathrm{~mol} \mathrm{~L}^{-1}$, and $1 \times 10^{-3} \mathrm{~mol} \mathrm{~L}^{-1}$. Rapidly mixing these THF solutions with large amount of water, which is a poor solvent for 1,7-TEG-PDI-C ${ }_{12}$, allows the observation of the concentration effects on the self-assembly morphology of 1,7TEG-PDI-C ${ }_{12}$.

The TEM images for the 1,7-TEG-PDI- $\mathrm{C}_{12}$ aggregates formed at the three concentrations are shown in Fig. 7. 1,7-TEG-PDI-C ${ }_{12}$ molecules self-assemble into thin nanofibers at the low concentration (Fig. 7a), nanotubes or nanosheet at the medium concentration (Fig. 7b), and nanoparticles at the high concentration (Fig. 7c). The enlarged images for the nanotube and the $\pi-\pi$ stacking in a nanoparticle are shown in Fig. 8a and b, respectively. The evidently different morphologies in Fig. 7 indicate the important role of concentration in the selfassembly behaviors of 1,7-TEG-PDI-C ${ }_{12}$. The UV-vis absorption spectra in Fig. 6 a show that the proportion of 1,7-TEG-PDI-C ${ }_{12}$ aggregates in THF solution increases with the solution concentration. Thus, when dispersed into water, 1,7-TEG-PDI$\mathrm{C}_{12}$ molecules in THF solution are more exposed to the surrounding water molecules at the lower solution
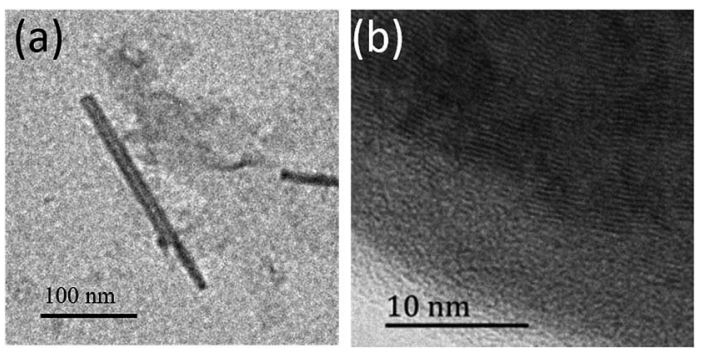

Fig. 8 The enlarged TEM images of (a) the nanotube in Fig. 7b and (b) the $\pi-\pi$ stacking in the nanosphere in Fig. $7 \mathrm{c}$.

concentrations than at the higher concentrations. This may be the reason why at the lower solution concentration $(1 \times$ $10^{-5} \mathrm{~mol} \mathrm{~L}^{-1}$ ), 1,7-TEG-PDI- $\mathrm{C}_{12}$ molecules in the less aggregated state formed thin nanofibers with more surface area exposed to the surrounding water molecules, whereas at the higher concentrations $\left(1 \times 10^{-4} \mathrm{~mol} \mathrm{~L}^{-1}\right.$, and $\left.1 \times 10^{-3} \mathrm{~mol} \mathrm{~L}^{-1}\right)$, the molecules assembled into nanotubes, nanoplates, and nanoparticles because of the increased proportion of the 1,7-TEGPDI-C $_{12} / 1,7-T E G-P D I-C_{12}$ aggregates, and the decreased proportion of the $1,7-\mathrm{TEG}-\mathrm{PDI}-\mathrm{C}_{12} /$ water interaction.

\section{Self-assemble morphology of 1,7-TEG-PDI-C ${ }_{12}$ under slow liquid-liquid diffusion}

To study the self-assembly morphology of $1,7-T E G-P D I-C_{12}$ developed under the slow THF/water mixing process, as illustrated in Fig. 9a, the THF solution of 1,7-TEG-PDI-C ${ }_{12}(1 \times$ $10^{-3} \mathrm{~mol} \mathrm{~L}^{-1}$ ) was carefully added to the top of the water to

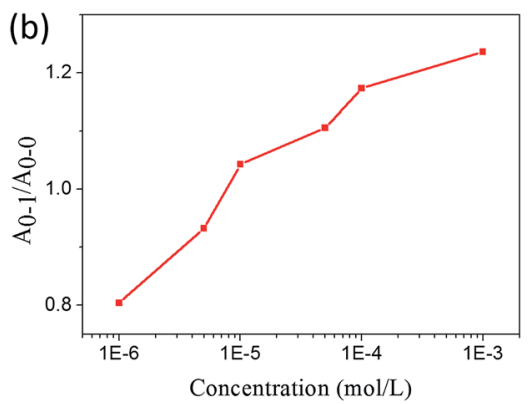

Fig. 6 (a) The UV-visible absorption spectra of the THF solutions of 1,7-TEG-PDI- $\mathrm{C}_{12}$ at different concentrations; (b) the concentration dependence of the $A_{0-1} / A_{0-0}$ ratio of the THF solutions.
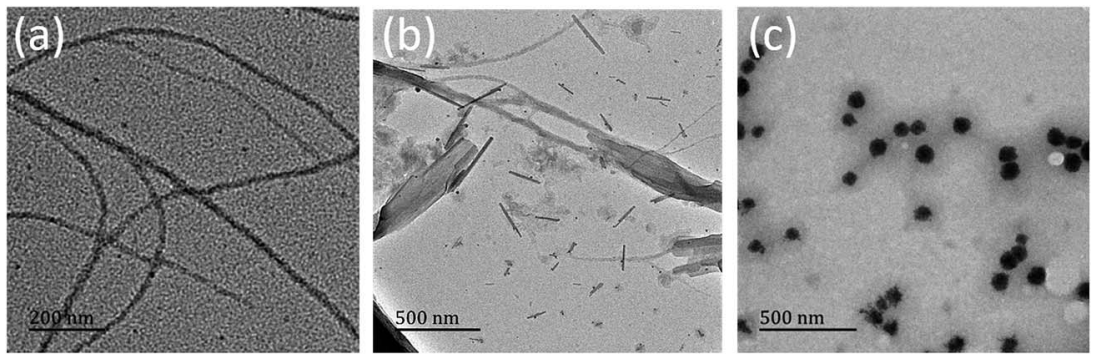

Fig. 7 TEM images of the self-assembled morphologies of 1,7-TEG-PDI- $\mathrm{C}_{12}$. The self-assembled morphologies were produced from the THF solutions of 1,7-TEG-PDI- $\mathrm{C}_{12}$ at concentrations of (a) $1 \times 10^{-5} \mathrm{~mol} \mathrm{~L}^{-1}$, (b) $1 \times 10^{-4} \mathrm{~mol} \mathrm{~L}^{-1}$, and (c) $1 \times 10^{-3} \mathrm{~mol} \mathrm{~L}^{-1}$. 
(a)

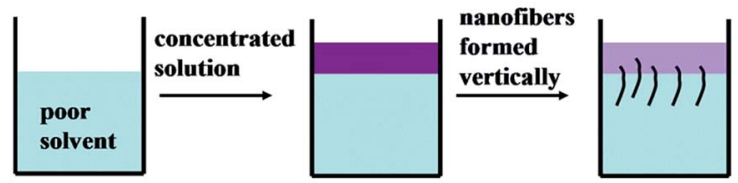

(b)

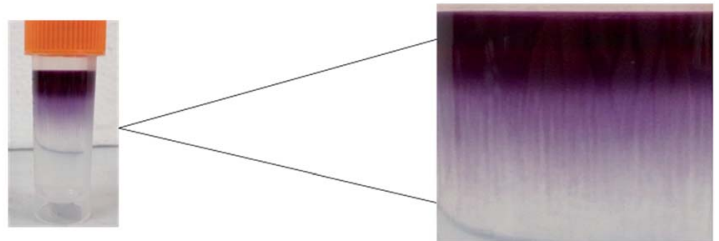

Fig. 9 (a) The illustration and (b) the experimental image of the thick nanofibers prepared via the slow solvent-diffusion of the THF solution of $1,7-T E G-P D I-C_{12}\left(1 \times 10^{-3} \mathrm{~mol} \mathrm{~L}^{-1}\right)$ into water.

allow the slow diffusion of the THF solution into water. As shown in Fig. 9b, purple fibers grow and align perpendicular to the THF/water interface, as the THF solution diffuse into the water. Compared to the rapid solution mixing method, which produces a powder of 1,7-TEG-PDI- $\mathrm{C}_{12}$, the slow liquid-liquid diffusion method gives solid sample of 1,7-TEG-PDI-C ${ }_{12}$ with larger sizes and more continuous morphology. The optical microscope (OM) image, and polarized light optical microscope (POM) image, and SEM images of the fibers are shown in Fig. 10. In Fig. 10a, the fibers show birefringence under POM, indicating that 1,7-TEG-PDI-C ${ }_{12}$ molecules form ordered packing in the fibers. The XRD pattern of the fibers is shown in Fig. 11. The four diffraction peaks at the lower $2 \theta$ angle have $d$ spacing of $34.9 \AA ̊ ㇒, 20.0 \AA ̊, 17.4 \AA$ A, and $13.1 \AA$, respectively. The

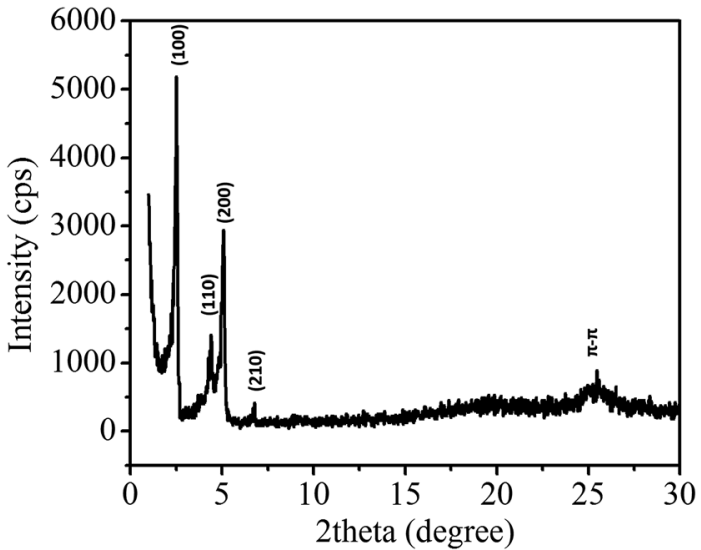

Fig. 11 XRD pattern of the 1,7-TEG-PDI- $C_{12}$ fibers prepared from the slow liquid-liquid diffusion method.

reciprocal ratio for the $d$-spacing of the peaks is $1: \sqrt{ } 3: \sqrt{ } 4: \sqrt{ } 7$, indicating that 1,7-TEG-PDI-C ${ }_{12}$ molecules pack into a hexagonal columnar phase with $a=b=40.3 \AA$, and $\gamma=120^{\circ}$. In addition, a broad diffraction hump with $d$-spacing of $3.49 \AA$, showing the $\pi-\pi$ stacking of the 1,7-TEG-PDI-C ${ }_{12}$ molecules, was also observed at the higher $2 \theta$ angle. The lateral dimension of the hexagonal lattice matches with the lateral dimension of a 1,7-TEG-PDI-C 12 molecule. Thus, it is confirmed that the purple fibers formed in the slow liquid-liquid diffusion process are constructed with the columns of 1,7-TEG-PDI-C ${ }_{12}$ molecules that are arranged in the hexagonal lattice.

In order to confirm whether or not intermolecular $\mathrm{H}$ bonding is present in 1,7-TEG-PDI- $\mathrm{C}_{12}$ aggregates, as shown in
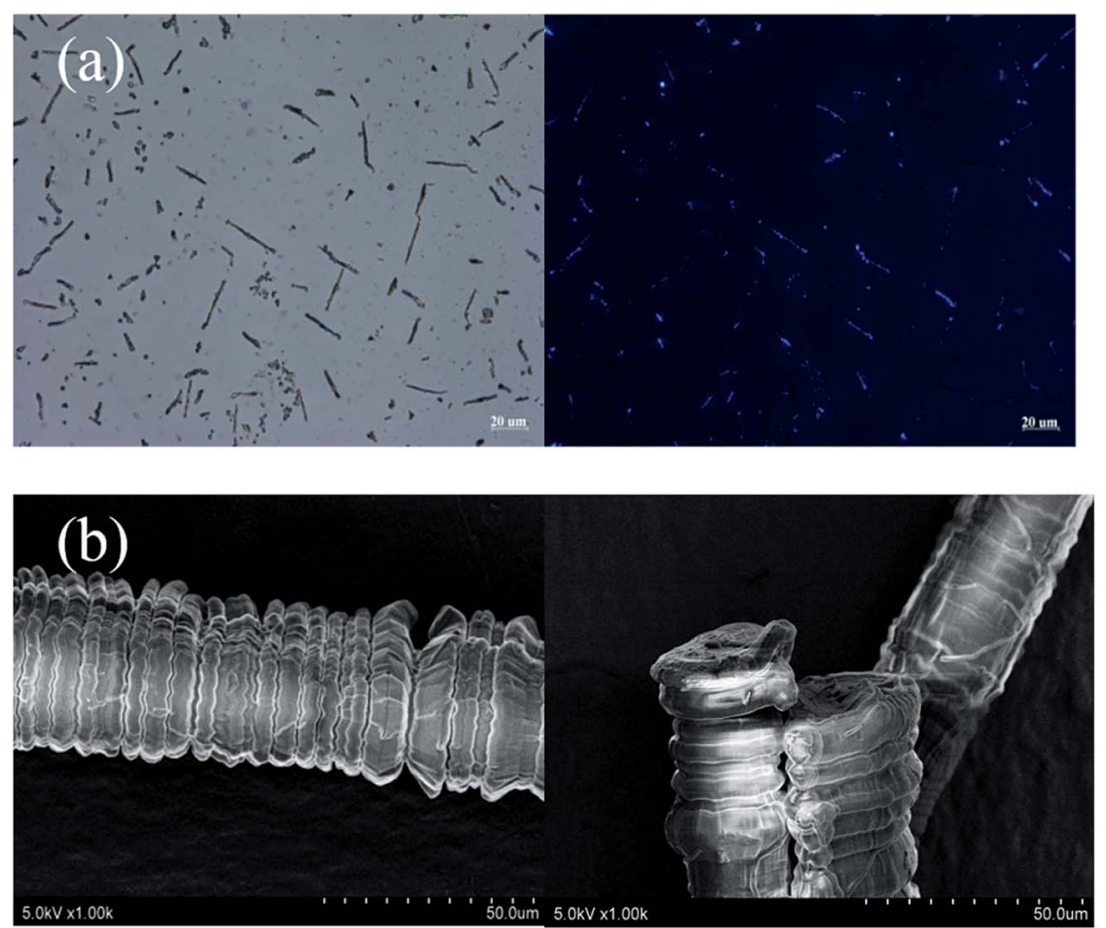

Fig. 10 (a) OM (left) and POM (right) images, and (b) SEM images of the 1,7-TEG-PDI- $\mathrm{C}_{12}$ fibers prepared from the slow liquid-liquid diffusion method. 
Fig. 12, the IR absorption spectra of 1,7-TEG-PDI-C ${ }_{12}$ were measured at $30{ }^{\circ} \mathrm{C}$ and $200{ }^{\circ} \mathrm{C}$, respectively. At $30{ }^{\circ} \mathrm{C}$, the absorption band at $3356 \mathrm{~cm}^{-1}$ indicating the presence of $\mathrm{H}^{-}$ bonding among the PDI molecules. The decrease of the absorption intensity at $3356 \mathrm{~cm}^{-1}$ and the increase of the wavenumber of the $\mathrm{C}=\mathrm{O}$ stretching at high temperature further confirmed that the intermolecular $\mathrm{H}$-bonds are disrupted by the enhanced thermal motion of 1,7-TEG-PDI-C ${ }_{12}$ at high temperature.

Since both $\pi-\pi$ interaction and hydrogen bonding are involved in the self-assembly process of 1,7-TEG-PDI-C ${ }_{12}$, a model intending to explain the concentration-dependent selfassembled morphologies of 1,7-TEG-PDI-C 12 was proposed and shown in Fig. 13. In the fast solution mixing process, the formation of the thin nanofibers at the lower solution concentration suggests that $\pi-\pi$ interaction works as the driving force for the formation of the $1 \mathrm{D}$ stacking of the 1,7-TEG-PDI- $\mathrm{C}_{12}$ molecules. The nanotubes, nanoplates and nanospheres were

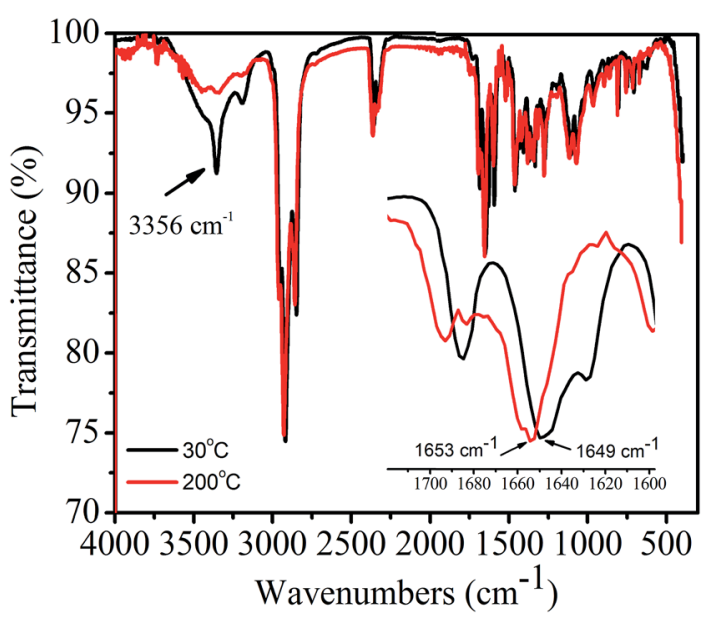

Fig. 12 FT-IR spectra of the 1,7-TEG-PDI- $C_{12}$ fiber at $30{ }^{\circ} \mathrm{C}$ and $200{ }^{\circ} \mathrm{C}$.

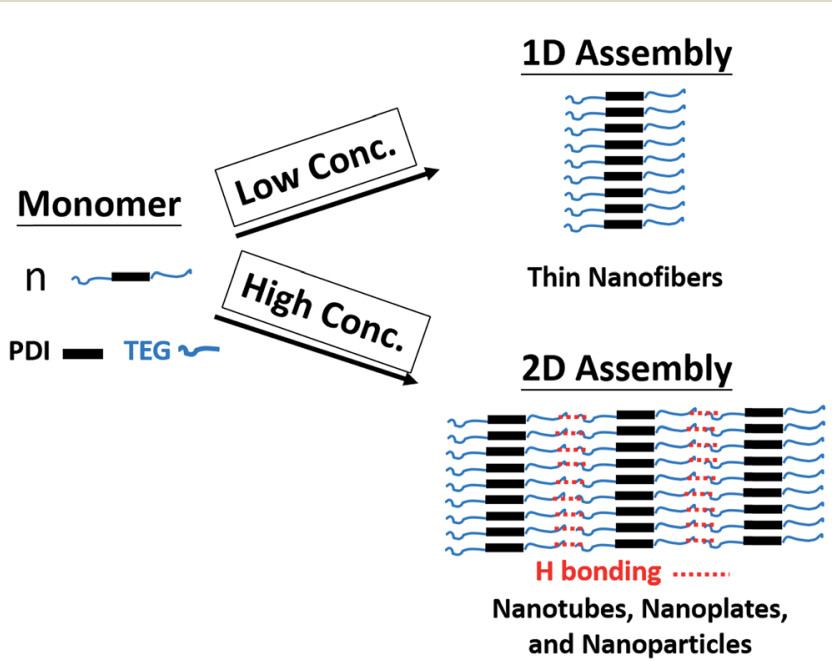

Fig. 13 Proposed mechanism for the concentration-dependent assembly behaviors of 1,7-TEG-PDI- $\mathrm{C}_{12}$. not observed at this concentration because the formation of $2 \mathrm{D}$ assembly structure requires additional 1,7-TEG-PDI-C 12 /1,7TEG-PDI- $\mathrm{C}_{12} \mathrm{H}$-bonding along the lateral direction. Therefore, the formation of the $2 \mathrm{D}$ assembly structures requires higher 1,7TEG-PDI-C $_{12}$ concentrations or slower liquid-liquid diffusion process, so that the 1,7-TEG-PDI- $\mathrm{C}_{12}$ molecules can either preaggregate in the THF solution, or have sufficient time to develop the lateral hydrogen bonds with their neighboring molecules to form the $2 \mathrm{D}$ structures. Otherwise, the thin nanofibers will be simply surrounded by water molecules and have only limited lateral dimensions. In Fig. 10b, another interesting feature of the 1,7-TEG-PDI-C ${ }_{12}$ fibers developed under slow liquid-liquid diffusion process is the uneven surface of fibers. The enlarged SEM image near the tip of a fiber shows that the macroscopic structure of the 1,7-TEG-PDI-C $\mathrm{C}_{12}$ fiber is similar to the shape of a Chinese herb, Cordyceps sinensis, or like a stack of flat plates, which have diameters around $15 \mu \mathrm{m}$. Such self-assembled morphology was not reported in the literature for the PDI derivatives. Mechanism regarding how the $\pi-$ $\pi$ interaction and $\mathrm{H}$-bonding of $1,7-\mathrm{TEG}-\mathrm{PDI}-\mathrm{C}_{12}$ synergetically assemble the molecules into this macroscopic structure will be further studied.

\section{Conclusions}

In this study, the synthesis and self-assembly behaviors of an amphiphilic PDI derivative, 1,7-TEG-PDI-C ${ }_{12}$, which contains -OH groups at its bay positions were studied. The difficulty in adding the bifunctional TEG substituents onto the bay position of 1,7dibrominated PDI in high yield was overcome by the careful choice of base, and the optimization of the reaction conditions. Under the synergetic effects of $\pi-\pi$ interaction and hydrogen bonding, 1,7-TEG-PDI-C ${ }_{12}$ demonstrated abundant concentrationdependent self-assembly morphologies. Nanofibers, nanotubes, nanoplates and nanospheres were observed form samples prepared from the solutions of 1,7-TEG-PDI-C ${ }_{12}$ at different concentrations. Although Yao and co-workers ${ }^{17}$ have observed that the amphiphilic PDI can self-assemble into nanoplates and nanoparticles in $\mathrm{MeOH} / \mathrm{H}_{2} \mathrm{O}$ solution, and Baram et al. ${ }^{15}$ have reported the self-assembled nanofibers and nanospheres of an amphiphilic bis-PDI derivative; the transformation of the selfassembled morphologies requires either dynamically protonating a 1,7-bis(pyridyloxyl)PDI derivative or reversible charging of the bis-PDI derivative. The roles of solution concentration and the bay-position -OH groups in the self-assembled morphology of amphiphilic PDI derivatives have never been investigated to the best of our knowledge. Our study reveals the importance of concentration and bay-position - $\mathrm{OH}$ groups in the formation of lateral TEG-PDI-C 12 /1,7-TEG-PDI-C ${ }_{12}$ hydrogen bonds, which are critical in the formation of $2 \mathrm{D}$ assembly morphology, and provides a facile physical methodology to manipulate the assembly morphology of amphiphilic PDI molecules.

\section{Acknowledgements}

This work was supported by the Program for Changjiang Scholars and Innovative Research Team in University 
(T2011079, IRT1221), Science and Technology Commission of Shanghai Municipality (16JC1400700).

\section{References}

1 S. S. Babu, V. K. Praveen and A. Ajayaghosh, Chem. Rev., 2014, 114, 1973-2129.

2 S. Chen, P. Slattum, C. Wang and L. Zang, Chem. Rev., 2015, 115, 11967-11998.

3 Y. Li, T. Liu, H. Liu, M. Z. Tian and Y. Li, Acc. Chem. Res., 2014, 47, 1186-1198.

4 F. Wurthner, Chem. Commun., 2004, 1564-1579.

5 P. D. Frischmann, K. Mahata and F. Wurthner, Chem. Soc. Rev., 2013, 42, 1847-1870.

6 Y. Shao, G.-Z. Yin, X. Ren, X. Zhang, J. Wang, K. Guo, X. Li, C. Wesdemiotis, W.-B. Zhang, S. Yang, M. Zhu and B. Sun, RSC Adv., 2017, 7, 6530-6537.

7 Y. Shao, X. Zhang, K. Liang, J. Wang, Y. Lin, S. Yang, W.-B. Zhang, S. Yang, M. Zhu and B. Sun, RSC Adv., 2017, 7, 16155-16162.

8 S. L. Wu, C. Y. Hong, K. Y. Wu, S. T. Lan, C. T. Hsieh, H. L. Chen and C. L. Wang, Chem.-Asian J., 2016, 11, 20112015.

9 W. W. Liang, C. F. Huang, K. Y. Wu, S. L. Wu, S. T. Chang, Y. J. Cheng and C. L. Wang, Chem. Sci., 2016, 7, 2768-2774.

10 D. Meng, D. Sun, C. Zhong, T. Liu, B. Fan, L. Huo, Y. Li, W. Jiang, H. Choi, T. Kim, J. Y. Kim, Y. Sun, Z. Wang and A. J. Heeger, J. Am. Chem. Soc., 2016, 138, 375-380.

11 Y. Huang, J. Wang, H. Zhai, L. Zhu and Z. Wei, Soft Matter, 2014, 10, 7920-7924.

12 K. Balakrishnan, A. Datar, T. Naddo, J. Huang, R. Oitker, M. Yen, J. Zhao and L. Zang, J. Am. Chem. Soc., 2006, 128, 7390-7398.
13 P. R. L. Malenfant, C. D. Dimitrakopoulos, J. D. Gelorme, L. L. Kosbar, T. O. Graham, A. Curioni and W. Andreoni, Appl. Phys. Lett., 2002, 80, 2517-2519.

14 P. Rajasingh, R. Cohen, E. Shirman, L. J. Shimon and B. Rybtchinski, J. Org. Chem., 2007, 72, 5973-5979.

15 J. Baram, E. Shirman, N. Ben-Shitrit, A. Ustinov, H. Weissman, I. Pinkas, S. G. Wolf and B. Rybtchinski, J. Am. Chem. Soc., 2008, 130, 14966-14967.

16 X. Zhang, Z. Chen and F. Würthner, J. Am. Chem. Soc., 2007, 129, 4886-4887.

17 Z. Zhang, C. Zhan, X. Zhang, S. Zhang, J. Huang, A. D. Li and J. Yao, Chem.-Eur. J., 2012, 18, 12305-12313.

18 Y. Chen, Y. Feng, J. Gao and M. Bouvet, J. Colloid Interface Sci., 2012, 368, 387-394.

19 L. Fan, Y. Xu and H. Tian, Tetrahedron Lett., 2005, 46, 44434447.

20 X. Zhang, S. Pang, Z. Zhang, X. Ding, S. Zhang, S. He and C. Zhan, Tetrahedron Lett., 2012, 53, 1094-1097.

21 D. Ke, C. Zhan, S. Xu, X. Ding, A. Peng, J. Sun, S. He, A. D. Li and J. Yao, J. Am. Chem. Soc., 2011, 133, 11022-11025.

22 Y. Tsarfati, V. Strauss, S. Kuhri, E. Krieg, H. Weissman, E. Shimoni, J. Baram, D. M. Guldi and B. Rybtchinski, J. Am. Chem. Soc., 2015, 137, 7429-7440.

23 G. Golubkov, H. Weissman, E. Shirman, S. G. Wolf, I. Pinkas and B. Rybtchinski, Angew. Chem., Int. Ed., 2009, 48, 926930.

24 A. E. Clark, C. Qin and A. D. Li, J. Am. Chem. Soc., 2007, 129, 7586-7595.

25 B. Fimmel, M. Son, Y. M. Sung, M. Grüne, B. Engels, D. Kim and F. Würthner, Chem.-Eur. J., 2015, 21, 615-630. 